\title{
社会竞争失败病因学的抑郁症树鼠模型
}

\author{
王 静 ${ }^{1,3}$, 周启心 ${ }^{1}$, 吕龙宝 ${ }^{2}$, 徐 林 $^{1}$, 杨跃雄 ${ }^{1, *}$ \\ (1. 中国科学院和云南省动物模型与人类疾病机理重点实验室, 中国科学院昆明动物研究所, 云南 昆明 $650223 ; 2$. 中国科学院昆明灵长类 \\ 研究中心, 中国科学院昆明动物研究所, 云南 昆明 650223;3. 中国科学院研究生院, 北京 100049)
}

摘要: 抑郁症是一种常见精神疾病, 主要表现为持续两周以上的情绪低落。世界卫生组织预测在 2030 年抑 郁症的疾病负担将高居所有疾病、伤残总负担的榜首。抑郁症面临三大难题: 1) 发病机理不完全清楚, 因而缺乏 有效的预测预防途径和生物学诊断；2) 现有单胺类抗抑郁症药物起效慢，也可能导致患者自杀风险增加；3) 缺乏 副作用小的非单胺类快速起效抗抑郁症药物。针对这三大难题, 长期以来, 应用抑郁症啮齿类模型的众多研究并 未取得实质性进展，至少部分因素归处于啮齿类与人类大脑功能的极大种属差异。树鼣是灵长类近亲，具有更接 近于人类的大脑功能。本文针对抑郁症发病机理假说、临床表象和抗抑郁症药物疗效等内容, 综述了社会竞争失 败病因学的抑郁症树獡模型可能会具有更好的疾病同源性、表象一致性和药物预见性。这一被长期忽视的抑郁症 树鼠模型尽管还需要进一步完善, 但对其进一步深入研究可能为解决抑郁症的三大难题提供了一条新途径。

关键词: 抑郁; 应激; HPA 轴; 药物代谢; 树鼠

中图分类号: Q175; Q133 文献标志码: A 文章编号: 0254-5853-(2012)01-0092-07

\section{A depression model of social defeat etiology using tree shrews}

\author{
WANG Jing ${ }^{1,3}$, ZHOU Qi-Xin $^{1}$, LÜ Long-Bao ${ }^{2}$, XU Lin ${ }^{1}$, YANG Yue-Xiong ${ }^{1, *}$ \\ (1. Key Laboratory of Animal Models and Human Disease Mechanisms, Chinese Academy of Sciences \& Yunnan Province, Kunming Institute of Zoology, \\ Kunming 650223, China; 2. Kunming Primate Research Center of the Chinese Academy of Sciences, Chinese Academy of Sciences \& Yunnan Province, \\ Kunming Institute of Zoology, Kunming 650223, China; 3. Graduate School of Chinese Academy of Sciences, Beijing 100049, China)
}

\begin{abstract}
Depression is a common neuropsychiatric disorder, marked by depressed mood for at least two weeks. The World Health Organization predicts that depression will be the number one leading cause of disease and injury burden by 2030. Clinical treatment faces at least three serious obstacles. First, the disease mechanism is not fully understood and thus there are no effective ways to predict and prevent depression and no biological method of diagnosis. Second, available antidepressants are based on monoamine mechanisms that commonly have a long delay of action and possibly cause a higher risk of suicide. Third, no other antidepressant mechanisms are available, with fast action and few side effects. Unfortunately, several decades of research based on rodent models of depression have not been successful in resolving these problems, at least partially due to the huge differences in brain function between rodents and people. Tree shrews are the closest sister to primates, and brain functions in these species are closer to those of humans. In this review, we discuss a tree shrew model of depression with social defeat etiology and aspects of construct, face and predicted validity of an animal model. Although a tree shrew model of depression has long been ignored and not fully established, its similarities to those aspects of depression in humans may open a new avenue to address this human condition.
\end{abstract}

Key words: Depression; Stress; HPA axis; Drug metabolism; Tree shrew

抑郁症是一类以持续的情绪低落、快感缺失为 主要临床特征的精神疾病 (David et al, 2011)。据世 界卫生组织估算, 2000 年精神疾病占全球疾病总负
担的 $12.3 \% ，$ 到 2020 年将上升到 15\%，至 2030 年抑 郁症将超过心脑血管、呼吸系统和恶性肿瘤等疾病, 居全球疾病总负担的榜首。在抑郁症的众多病因学

\footnotetext{
收稿日期: 2011-11-16; 接受日期: 2012-01-19

基金项目：国家自然科学基金（81171294）; 中科院知识创新工程重要方向项目（KSCX2-EW-R-12, KSCX2-EW-J-23）

*通信作者(Corresponding author), E-mail: yangyx@mail.kiz.ac.cn

第一作者: 王静, 博士研究生, 主要研究方向为树鼠的精神疾病动物模型创制和机理研究。E-mail:wangjing0917@gmail.com
} 
研究中可分为三大类: 遗传与发育因素、反复发作 的躯体疾患、神经化学与激素障碍。在这些病因学 中，应激损伤至少占据了 $50 \%$ 的贡献(Cohen et al, 2007)。然而, 目前尚缺乏理想的动物模型能使我 们获得病因学的确切证据, 现阶段抑郁症的疾病模 型还有许多需要改进的地方。本文分析了多种抑郁 症疾病模型建立的方法和有效性, 对未来可能发 展的抑郁模型提出设想, 为新型模型的开发和完 善提供思路和方法, 以期为更好地研究抑郁症提供 依据。

\section{1 抑有症机理与动物模型}

\section{1 抑郁症发生机理假说}

至今抑郁症的发生机理仍然不清楚，目前对抑 郁症的发病机理有以下几种假说: “单胺类递质” 假说、“神经营养缺乏” 假说、“海马神经元受损” 假说和 “应激”假说。其中，“单胺类递质”假说 为目前大多数人接受的理论, 基于单胺类递质的药 物至今仍是抑郁症治疗的一线药物 (Berton \& Nestler, 2006; Naughton et al, 2000)。但是，目前对抑 郁症的临床治疗并不十分理想，药物治疗对约 30\% 的患者无效，约 $50 \%$ 的服药患者仍反复发作，这些 抗抑郁症药物几乎都靶向增强神经递质 5-着色胺 (5-HT)和去甲肾上腺(NE)系统功能, 至少需要 2-4 周连续服药才能部分地改善或控制抑郁症症状, 且 可能导致患者自杀风险增加。随后的研究进展表明, 抑郁症的发生机理远比 “单胺递质” 假说更加复杂 (Den Boer \& Westenberg, 1988; Heninger et al, 1996)。 经典的 “单胺递质” 假说无法解释单胺类抗抑 郁药不能改善所有抑郁症患者的症状以及单胺类 抗抑郁药物的起效时间延长的现象, 提示存在 “单 胺递质” 假说之外的其他抑郁症发病机制。随着研 究的深入，人们提出了抑郁症发病机制中的 “神经 营养缺乏” 假说。脑源性神经营养因子(BDNF)是成 年哺乳动物中枢神经系统分布最广、含量最高的神 经营养因子，对神经元的存活、分化、神经再生，突 触传递和突触可塑性具有重要作用, 大量研究表明 BDNF 参与抑郁症的发生、发展过程。临床研究发 现未进行抗抑郁治疗的重症抑郁症患者和抑郁性 格表现的志愿者血中 BDNF 浓度比正常健康志愿者 低(Karege et al, 2005; Lee et al, 2007), 并且这种低 水平的 BDNF 可以通过抗抑郁治疗得到纠正 (Gervasoni et al, 2005; Shimizu et al, 2003)。在动物
模型中脑内注射 BDNF 也可以逆转抑郁样行为 (Krishnan \& Nestler, 2008)。由此提出了抑有症发病 机制中的 “神经营养因子” 假说，认为脑源性神经 营养因子缺乏导致抑郁症的发生，而增加脑内脑源 性神经营养因子的表达有抗抑郁作用。

同时，越来越多的证据表明神经可塑性异常及 海马神经再生障碍可能是重症抑郁的重要神经生 物学基础之一(Castrén, 2005; Duman, 2004; Manji et $\mathrm{al}, 2001)$, 故而提出了抑郁症的 “海马神经元受损” 假说。众多研究从不同角度提示神经新生在抑郁发 生、发展以及治疗中发挥重要生理功能(Cole et al, 2010; Colla et al, 2007; Santarelli et al, 2003), 海马 齿状回神经新生已经成为治疗抑郁症的热点之一, 但是抑郁症神经新生异常的机理、抗抑郁药促进神 经元新生的调控等有待深入研究。

另外, 临床和流行病学研究发现应激是抑郁症 发生的重要危险因素。应激是指人或动物在生理上 的适应和心理上的激活，是体内的警示系统，只要 在期望与事实之间存在矛盾就会引发应激事件 (Johnson \& Sarason, 1978; Pittenger \& Duman, 2008)。应激反应是人类生存所必须的，过高或过低 的应激水平均不利于机体存活及发展，适度应激本 身无害也不会引起疾病, 但是长期持续处于高应激 状态时, 身体和大脑的稳态会受到威胁而产生精神 疾病(de Kloet et al, 2005)。因此应激动物模型是目 前研究抑郁症发病机制的一种常用实验动物模型。

\section{2 抑有症常用模型——啮齿类动物模型}

目前最常用的动物模型多是基于 “应激” 假说 而建立，以啮齿类动物抑郁症模型为主(Duman \& Monteggia, 2006)。最常用的应激模型是慢性不可预 知温和应激模型(chronic unpredictable mild stress, CUMS), 其中包含多种应激刺激, 以动物不可预见 的顺序出现(Grønli et al, 2004)。除 CUMS 模型外, 还有以失去回避能力为指标的习得性绝望模型 (Vaugeois et al, 2004; Willner, 2005)。最近还发展了 慢性睡眠剥夺、持续黑暗后用强迫游泳和悬尾作为 检测方法的抑郁模型(Gonzalez \& Aston-Jones, 2008; Wilson, 2002)。另外, 强迫游泳还可以作为一种慢 性应激，通过每天游泳 10 分钟，连续 1 周造模，来 评价抗抑郁药起效的时间点。

基于 “单胺类递质” 假说建立的模型有以体温 为指标的利血平单胺类递质耗竭模型(Baumeister et al, 2003; Skalisz et al, 2002)。除此之外还有通过药物 
诱导、人为因素作用而发展的抑郁模型, 例如通过 嗅球切除、免疫刺激(毒素、炎症因子)和兴奋剂刺 激(安非他明、摇头丸) 等因素诱发抑郁症模型 (Dandekar et al, 2009; Holmes et al, 2002; Slotkin \& Seidler, 2006; Túnez et al, 2010)。此外, 科学家们利 用啮齿类动物发展了以旷场中的自发运动量为指 标评价运动抑制的旷场行为模型(Airan et al, 2007; Broderick, 2002)。

尽管这些抑郁症啮齿类模型在抑郁症的发病 机理研究和抗抑郁症新药的研发中已经发挥了巨 大作用，临床抗抑郁症药物的疗效清楚表明，这些 模型存在不可克服的缺陷。都只能部分模拟人类抑 郁症的核心症状, 还没有一种动物模型可以完全模 拟抑郁症的所有表现, 而且每个模型都还不能完全 排除环境、心理和遗传因素等交叉影响。例如，应 用最广泛的 CUMS 模型虽然可以较好地模拟抑郁 症发生的环境诱因和临床上的病理进程, 致使动物 的行为改变、血浆皮质酮升高等均与内源性抑郁症 状相似, 维持时间长, 且经典抗抑郁药治疗有效。 但该模型涉及的应激因子众多，使得人们难于获得 病因学上的确切证据。严重地削弱了该动物模型的 结构效度。

虽然目前已经有众多抑郁症动物模型, 然而, 回顾近年来抗抑郁药物研究的发展, 发现现有临 床抗抑郁药物均未克服普遍存在的疗效延迟缺陷。 有报道显示在抗抑郁药物治疗最初的数周里, 尝 试自杀的风险远高于以后的时期 (Simon, 2006)。因 而, 抗抑郁药物的快速起效极其重要, 可以预见 这将成为今后抗抑郁药物研发的主要方向。建立对 快速抗抑郁作用敏感、性状稳定可靠、操作性强且 对具体症状有可鉴别性的动物模型，非常有必要。

一般动物模型应当满足三个一致性验证：(1) 疾病同源性(construct validity), 即与人类疾病具有 相似的发病机制; (2)表象一致性(face validity), 即 与人类疾病具有相似的行为表象; (3)药物预见性 (predictive validity), 即与人类疾病具有相似的药物 治疗反应(Willner, 1991; Xu, 2011)。一个好的抑郁 症动物模型应该具有好的药效预测性, 行为表现的 模拟性，药物作用时间也应当接近临床，受其它药 物干扰少, 有合理的理论基础, 行为学改变和内分 泌改变持续时间足够长等特点。而啮齿类与人类之 间存在极大的种属差异, 还不能令人满意地满足上 述需求, 因而需要建立非人灵长类动物模型进行补
充。

值得一提的是，近年来发展的社会竞争失败 (social defeat)动物模型, 能较好地再现人类在生活 日趋激烈的竞争和生活压力中接触应激性生活事 件的方式，和情绪持续低落、沉重的社会挫败感和 有罪感、乐趣丧失等抑郁症核心临床症状的心理过 程。且应激因子单一，有利于病因学分析。但啮齿 类固有的群居方式，导致建立社会竞争失败抑郁症 模型无法获得稳定的结构效度, 而非人灵长类动物 的近亲树鼣有可能克服这一缺陷。

\section{2 树鼠抑郁症的社会竞争失败模型}

\section{1 树鼠句可作为模式动物的潜在优势}

对树鼠的研究可以追溯到 1780 年。起初由于 对其分类上的不确定而把树鼣定义为 “松鼠” (squirrels), 至今这种混淆仍然存在。大约 80 年前有 种种报道称树鼠与灵长类相似, Le Gros Clark (1924) 以脑解剖为基础研究发现现代树鼠与灵长类之间 有直接的系统发生关系。之后有人质疑这种系统发 生关系，而将树鼣排除在灵长类之外。随后几十年 对树鼣及其系统发生学关系研究成为热点。如今, 树鼠被单独列为一目一一树鼠目(Scandentia), 基于 最近分子系统发生学的研究, 树鼠目作为一个分支 位于灵长目和食虫目之间(Fuchs \& Corbach-Söhlef, 2010)。

树鼣科分为两个亚科: 其中, 昼行性树獡亚科 有 5 个属(Tupaia, Anathana, Dendrogale, Lyonogale, Urogale); 夜行性树鼠亚科则只有笔尾树鼠属 (Ptilocercus)。从印度到菲律宾, 从中国西南到爪哇 岛、婆罗洲、苏门答腊岛、巴厘岛都有树舄分布，热 带雨林以及热带、亚热带地区都是其自然栖息地 (Fuchs \& Corbach-Söhlef, 2010)。我国所拥有的树鼠 (Tupaia belangeri chinensis)资源主要分布于云南、 海南、西藏和贵州等省，均为昼行性。

虽然树鼣种属间有不同之处，但是它们也有共 性: 都是体型较小、敏捷、杂食, 喜爱水果和无脊 椎动物, 特别是节肢动物。从树上 (Dendrogale, Tupaia minor, Ptilocercus) 到 陆地 (Lyonogale, Urogale)都是树鼠的活动范围, 但更多是在陆地找 食。缅甸树鼠(Tupaia belangeri)的视觉系统发育很 好且可辨别颜色。对其的声学研究发现，树鼠没有 超声波, 但可发出 8 种不同的声音, 频率范围在 $(0.4 \sim 20) \mathrm{kHz}$ 之间，其中，有 4 种的功能是用来表 
达警告、注意以及防御等含义(Kirchhof et al, 2001)。 树鼠体形小 $(200 \sim 250 \mathrm{~g})$ 、繁殖和生长周期短、 每胎 4 6 只后代、近亲繁殖能产生稳定后代, 完全 有可能建立标准化品系。作为新型抑郁症动物模型, 树鼠与啮齿类相比, 有许多灵长类特有表型 (Zambello et al, 2010), 并且由于对应激极度敏感和 雄性间好斗的特点, 特别适合于应激相关的抑有症 模型建立、机理研究和新药研发。树鼠作为抑郁症 模式动物的潜在优势主要有: (1) 大脑前额叶皮质 发育良好与丘脑之间形成双向投射(Divac et al, 1978); (2) 在脑一应激回路下丘脑一垂体一肾上腺 (hypothalamic-pituitary-adrenal axis, HPA)轴中, 皮 质醇是激动糖皮质激素受体的主要成份 $(\mathrm{Ohl}$ et $\mathrm{al}$, 1999), 与人类极其类似, 而在大鼠, 则主要为皮质 酮; (3) 社会竞争失败后出现睡眠紊乱、运动抑制、 情绪持续低落甚至绝食死亡等(Legros et al, 2007), 与人类抑郁症表象非常类似。(4) 社会竞争失败可 导致树獡的海马神经细胞萎缩, 其机理与人类的 HPA 轴过度激活和 5-HT1A 受体表达下调、抑制海 马神经元新生等有关(Czéh et al, 2006), 也与人类抑 郁症机理非常类似。(5) 树鼠的糖皮质激素和盐皮 质激素受体, 促肾上腺激素释放激素 $(\mathrm{CRH})$ 受体, 以及 $\mathrm{a} 2 \mathrm{~A}$-肾上腺受体 DNA 序列与人类相应的基因 有很高的同源性，高达 $90 \% \sim 98 \%$, 而大鼠只有 $80 \%$ (van Kampen et al, 2002)。(6) 正常的节律是健 康的重要保障, 在研究睡眠失调与抑郁症之间的关 系以及抗抑郁药物对昼夜节律的影响中, 昼行性动 物与夜行性动物相比无疑具有更大的优势 (Kronfeld-Schor \& Einat, 2012)。而树鼠可以弥补啮 齿类动物模型这个天生的缺陷。这些特点表明抑郁 症树鼣模型也许能很好模拟人类抑有症, 为抑郁症 的疾病机理研究、抗抑郁症新药研发, 为发展早期 诊断技术和预防措施等提供理想的或有显著特色 和优势的动物模型。

\section{2 树鼠抑郁症社会竞争失败模型的疾病同源性}

疾病同源性要求动物模型与人类疾病具有相 似的发病机理。人类的服从行为主要与低自尊心相 关, 这点在抑郁症评价中属于较难评价的因子 (Brown et al, 1990)。树鼠具有很强的社会属性, 特 别是雄性具有很强的领地意识。无论是野生还是实 验环境下, 都可以观察到树鼠的领地标记行为, 如 有同种物种入侵会发生打斗(Eichmann \& Holst, 1999)。这种由社会竞争失败导致的社会遭遇在一定
程度上可以模拟人类面对的自尊丧失。而啮齿类虽 然在一定程度上也有社会等级制度，但是由于长期 的实验室群养驯化, 这种社会属性逐渐被弱化, 因 而很难建立社会竞争失败病因学抑郁模型。而 CUMS 虽然已被广泛用来研究应激诱发的抑郁症 机理, 但是由于这些应激多为人为产生, 不是动物 自发产生的, 导致疾病同源性较差, 进而导致抑郁 症机理研究缓慢。

实验室建立的树鼠社会竞争失败抑郁症模型 通过自发打斗, 使其中一只遭遇社会竞争失败而成 为从属者, 其活动性降低、躲避行为增加、进食量 减少等表现(Wang et al, 2011)在一定程度上都可以 模仿人类的低自尊心。在人类社会失去社会地位被 认为是一种应激生活事件, 从而导致患抑郁症风险 增加。因此树鼠由应激诱导的抑郁症模型与啮齿类 相比更接近人类抑郁症的发病起因, 推测可能具有 相似的发病机理。

\section{3 树鼠抑有症社会竞争失败模型的表象一致性}

近几年研究表明利用树鼠由社会竞争失败诱 导的抑郁症模型来研究应激相关疾病的发病机理 更合适、更自然。经过社会竞争失败, 被攻击后的 树舀长期与主导地位的树䳔保持视觉、嗅觉、听觉 上的接触, 其行为、生理和神经内分泌均会发生显 著改变(Fuchs, 2005)。通过定量的行为学分析发现, 从属树鼣趋向于避开主导者的视野范围, 减少活动 量, 停止自我梳理行为。并且从属者的生理节律被 严重破坏, 主要表现为早醒。其体重的减少主要由 摄食取水减少以及代谢率增加引起。对从属者进行 内分泌参数分析发现，肾上腺皮质激素皮质醇浓度 以及肾上腺的重量持续增长, 与此同时其生殖腺功 能降低。对从属树鼠的脑进行研究后的结果表明, 5-HT1A 受体减少, a2-肾上腺受体功能下调。海马在 调节 HPA 轴的负反馈(Herman et al, 1993)、空间信 息处理和学习记忆方面(Shen et al, 2004)发挥着重 要作用。糖皮质激素、盐皮质激素以及促肾上腺激 素释放激素受体下调可改变雉体神经元结构并减 缓神经发生, 进而影响 HPA 轴的活动(Meyer et al, 2001)。对上述由应激直接诱导的从属树鼠行为、生 理、中枢神经改变的认知解释, 只能是在长期处于 同种主导者影响下而产生。另外, 与从属者表现相 反, 主导者无明显的行为、生理改变(Fuchs et al, 1995)。

从属树鼠的这些行为学、神经内分泌变化, 与 
对啮齿类或灵长类注射促肾上腺激素释放激素 $(\mathrm{CRH})$ 来模拟抑郁样症状表现一致, 重要的是其表 现与抑郁症患者观察到的症状相一致(Spitzer et al, 1994)。尽管必须承认情感障碍的关键症状(比如抑 郁情绪、无精打采甚至自杀等)都是由主观产生的、 且无法在啮齿类动物模型上进行模拟, 但是, 在从 属树鼠行为反应中可以观察到抑郁症患者的上述 情感症状。因此, 树鼠由应激诱导的抑有症模型拥 有抑郁症的表象一致性。

\section{4 树鼠抑郁症社会竞争失败模型的药物预见性}

药物预见性是指在动物模型上有效的药物可 预见在临床上也会有效的能力, 是评价动物模型是 否真正模拟人类疾病最关键的指标。Fuchs et al (1996)采用口服氯咪帕明的方式, 比较抑郁症患者 和社会竞争失败抑郁症模型的树鼠服用抗抑郁症 药物的异同, 为了获得抗抑郁药的真实情况, 在每 日用药的同时, 应激因素仍然存在, 氯咪帕明的治 疗贯穿 4 周的临床关键期。研究结果表明, 三环类 抗抑郁药氯咪帕明对从属树鼠有效。

人类氯咪帕明有效剂量是使氯咪帕明和去甲 氯咪帕明的血清浓度在 $230 \sim 450 \mathrm{ng} / \mathrm{mL}$ 范围内。经 高效液相色谱(high-performance liquid chromatography, HPLC)验证确定树鼠连续 5 天每天口服大约 50 $\mathrm{mg} / \mathrm{kg}$ 可以导致氯咪帕明和去甲氯咪帕明血清浓度 达到 $240 \mathrm{ng} / \mathrm{mL}$, 这是两种复合物血清中的最低浓 度(Gex-Fabry et al, 1999)。 5 天后树鼠氯咪帕明的代 谢物主要有去甲氯咪帕明、8-羟基氯咪帕明、10羟基去甲氯咪帕明和 8-羟基去甲氯咪帕明。对其进 行测试, 并与每天口服 $150 \mathrm{mg}$ 氯咪帕明的抑有症 患者的血清代谢物相比, 发现树鼠与人类氯咪帕明 代谢途径相似(van Kampen et al, 2002)。但是在大鼠 $(20 \mathrm{mg} / \mathrm{kg})$ 的血清中只有去甲氯咪帕明, 而没有 8-或 10-美基代谢物(Weigmann et al, 1998)。

对抑有症患者的持续观察发现，抑郁症患者的 HPA 轴功能紊乱, 用三环类抗抑有症药可以恢复从 属树鼠 HPA 轴的活动。这可能是由于药物直接作 用于相关脑区的 5-HT 和去甲肾上腺素循环, 进而 调节具有调节由垂体产生的促肾上腺皮质激素 $(\mathrm{ACTH})$ 和其他类吗啡样神经肽的合成与释放作用 的 CRF (Corticotropin release factor) 系统 (Nikolarakis et al, 1987)。
对人类和动物的研究报道结果表明, 氯咪帕明 除了有抗抑郁作用还有抗焦虑作用。对苯二氮受体 拮抗剂地西泮在从属动物活动性、标记行为以及尿 液皮质醇等指标上的作用研究表明, 地西泮不起作 用从而间接证明氯咪帕明起抗抑郁作用, 树鼣社会 竞争失败模型是抑郁症模型(van Kampen et al, 2000)。由此可见，抑制 5-HT 重摄取的三环类抗抑 郁药氯咪帕明对受心理社会应激的树鼠具有疗效, 树鼠社会竞争失败的抑郁症模型具有药物预见性。

\section{3 结论与展望}

抑郁症是情绪、思维等脑高级功能紊乱。这些 脑高级功能很难在抑有症啮齿类模型中明确体现。 例如人类抑有症患者常见的自杀行为, 以及各种表 情特征。新药研究表明, 啮齿类中的实验结果在人 类临床中重现的概率约为万分之一, 导致了新药研 发的困难和人力物力浪费。其根本原因在于啮齿类 与人类之间存在巨大的种属差异, 导致啮齿类动物 模型的药物预见性很差。树鼠被认为是低等灵长类 或与灵长类有共同祖先, 强烈应激可导致树鼣绝食 甚至死亡，从而可以模拟人类的自杀行为。同时在 我们实验观察中发现, 树䳔具有一定的表情, 为研 究人类抑郁症患者的各种表情特征提供可能性, 使 其成为一种研究人类抑郁症较为理想的动物模型。

上文提到受应激树舄主要表现为体重下降、活 动量减少、标记行为减少以及内分泌失调, 海马神 经元减少等, 与临床结果基本一致。但是, 仅仅知 道动物患有抑有还远远不够, 抑郁程度评估对抑郁 的早期预防具有重要意义。在临床上对抑郁的严重 度评估主要依靠抑郁症量表, 例如汉密尔顿抑郁量 表(Hamilton depression scale, HAMD), 即通过患者 的得分即可判断其抑郁程度。目前, 还没有一个合 适的、用来判断动物抑郁程度的类似量表。因此, 需 要在今后的研究中通过与现有的应激病因学抑郁 症啮齿类模型、有应激症状或有应激生活史的人类 抑有症数据进行比较, 归纳出抑郁症树䳔模型, 既 区别于抑郁症啮齿类模型，又类似于人类抑郁症的 特征, 进而创制出一个适合于树鼣的抑郁症量表。 这将为阐明与人类抑郁症之间的相似性以及抑郁 症早期预防、发病机理研究和抗抑郁症新药研发提 供重要依据。 


\section{参考文献:}

Airan RD, Meltzer LA, Roy M, Gong YQ, Chen H, Deisseroth K. 2007. High-speed imaging reveals neurophysiological links to behavior in an animal model of depression[J]. Science, 317(5839): 819-823.

Baumeister AA, Hawkins MF, Uzelac SM. 2003. The myth of reserpine-induced depression: role in the historical development of the monoamine hypothesis[J]. J Hist Neurosci, 12(2): 207-220.

Berton O, Nestler EJ. 2006. New approaches to antidepressant drug discovery: beyond monoamines[J]. Nat Rev Neurosci, 7(2): 137-151.

Broderick PA. 2002. Interleukin $1 \alpha$ alters hippocampal serotonin and norepinephrine release during open-field behavior in Sprague-Dawley animals: differences from the Fawn-Hooded animal model of depression[J]. Prog Neuropsychopharmacol Biol Psychiatry, 26(7-8): 1355-1372.

Brown GW, Bifulco A, Veiel HOF, Andrews B. 1990. Self-esteem and depression. II. Social correlates of self-esteem[J]. Soc Psychiatry Psychiatr Epidemiol, 25(5): 225-234.

Castrén E. 2005. Is mood chemistry?[J]. Nat Rev Neurosci, 6(3): 241-246.

Cohen S, Janicki-Deverts D, Miller GE. 2007. Psychological stress and disease[J]. JAMA, 298(14): 1685-1687.

Cole J, Toga AW, Hojatkashani C, Thompson P, Costafreda SG, Cleare AJ, Williams SCR, Bullmore ET, Scott JL, Mitterschiffthaler MT, Walsh ND, Donaldson C, Mirza M, Marquand A, Nosarti C, McGuffin P, Fu CHY. 2010. Subregional hippocampal deformations in major depressive disorder[J]. J Affect Disord, 126(1-2): 272-277.

Colla M, Kronenberg G, Deuschle M, Meichel K, Hagen T, Bohrer M, Heuser I. 2007. Hippocampal volume reduction and HPA-system activity in major depression[J]. J Psychiatr Res, 41(7): 553-560.

Czéh B, Simon M, Schmelting B, Hiemke C, Fuchs E. 2006. Astroglial plasticity in the hippocampus is affected by chronic psychosocial stress and concomitant fluoxetine treatment[J]. Neuropsychopharmacology, 31(8): 1616-1626.

Dandekar MP, Singru PS, Kokare DM, Subhedar NK. 2009. Cocaine- and amphetamine-regulated transcript peptide plays a role in the manifestation of depression: social isolation and olfactory bulbectomy models reveal unifying principles[J]. Neuropsychopharmacology, 34(5): 1288-1300.

David H, Barlow V, Mark Durand. 2011. Abnormal Psychology: An Integrative Approach 6th Ed[M]. USA: Wadsworth.

de Kloet ER, Joëls M, Holsboer F. 2005. Stress and the brain: from adaptation to disease[J]. Nat Rev Neurosci, 6(6): 463-475.

Den Boer JA, Westenberg HGM. 1988. Effect of a serotonin and noradrenaline uptake inhibitor in panic disorder; a double-blind comparative study with fluvoxamine and maprotiline[J]. Int Clin Psychopharmacol, 3(1): 59-74.

Divac I, Björklund A, Lindvall O, Passingham RE. 1978. Converging projections from the mediodorsal thalamic nucleus and mesencephalic dopaminergic neurons to the neocortex in three species[J]. J Comp Neurol, 180(1): 59-71

Duman RS. 2004. Depression: a case of neuronal life and death?[J]. Biol Psychiatry, 56(3): 140-145.

Duman RS, Monteggia LM. 2006. A neurotrophic model for stress-related mood disorders[J]. Biol Psychiatry, 59(12): 1116-1127.

Eichmann F, Holst DV. 1999. Organization of territorial marking behavior by testosterone during puberty in male tree shrews[J]. Physiol Behav, 65(4-5): 785-791.

Fuchs E. 2005. Social stress in tree shrews as an animal model of depression: An example of a behavioral model of a CNS disorder[J]. CNS Spectr, 10(3): 182-190.
Fuchs E, Corbach-Söhle S. 2010. Tree Shrew[M]. The UFAW Handbook on the Care and Management of Laboratory and Other Research Animals. Eighth Edition. Singapore: Robert Hubrecht and James Kirkwood, 262-275.

Fuchs E, Kramer M, Hermes B, Netter P, Hiemke C. 1996. Psychosocial stress in tree shrews: clomipramine counteracts behavioral and endocrine changes[J]. Pharmacol Biochem Behav, 54(1): 219-228.

Fuchs E, Uno H, Flügge G. 1995. Chronic psychosocial stress induces morphological alterations in hippocampal pyramidal neurons of the tree shrew[J]. Brain Res, 673(2): 275-282.

Gervasoni N, Aubry JM, Bondolfi G, Osiek C, Schwald M, Bertschy G, Karege F. 2005. Partial normalization of serum brain-derived neurotrophic factor in remitted patients after a major depressive episode[J]. Neuropsychobiology, 51(4): 234-238.

Gex-Fabry M, Balant-Gorgia AE, Balant LP. 1999. Clomipramine concentration as a predictor of delayed response: a naturalistic study $[\mathrm{J}]$. Eur J Clin Pharmacol, 54(12): 895-902.

Gonzalez MMC, Aston-Jones G. 2008. Light deprivation damages monoamine neurons and produces a depressive behavioral phenotype in rats[J]. Proc Natl Acad Sci USA, 105(12): 4898-4903.

Grønli J, Murison R, Bjorvatn B, Sørensen E, Portas CM, Ursin R. 2004 Chronic mild stress affects sucrose intake and sleep in rats[J]. Behav Brain Res, 150(1-2): 139-147.

Heninger GR, Delgado PL, Charney DS. 1996. The revised monoamine theory of depression: a modulatory role for monoamines, based on new findings from monoamine depletion experiments in humans[J]. Pharmacopsychiatry, 29(1): 2-11.

Herman JP, Watson SJ, Chao HM, Coirini H, McEwen BS. 1993. Diurnal regulation of glucocorticoid receptor and mineralocorticoid receptor mRNAs in rat hippocampus[J]. Mol Cell Neurosci, 4(2): 181-190.

Holmes PV, Masini CV, Primeaux SD, Garrett JL, Zellner A, Stogner KS, Duncan AA, Crystal JD. 2002. Intravenous self-administration of amphetamine is increased in a rat model of depression[J]. Synapse, 46(1): 4-10.

Janečka JE, Miller W, Pringle TH, Wiens F, Zitzmann A, Helgen KM, Springer MS, Murphy WJ. 2007. Molecular and genomic data identify the closest living relative of primates[J]. Science, 318(5851): 792-794.

Johnson JH, Sarason IG. 1978. Life stress, depression and anxiety: internal-external control as a moderator variable[J]. J Psychosom Res, 22(3): 205-208

Karege F, Bondolfi G, Gervasoni N, Schwald M, Aubry JM, Bertschy G. 2005. Low brain-derived neurotrophic factor (BDNF) levels in serum of depressed patients probably results from lowered platelet BDNF release unrelated to platelet reactivity[J]. Biol Psychiatry, 57(9): 1068-1072.

Kirchhof J, Hammerschmidt K, Fuchs E. 2001. Aggression and dominance in tree shrews (Tupaia belangeri)[M]. Prevention and control of aggression and the impact on its victims. New York: Kluwer Academic/Plenum Publishers, 409-414.

Krishnan V, Nestler EJ. 2008. The molecular neurobiology of depression[J]. Nature, 455(7215): 894-902.

Kronfeld-Schor N, Einat H. 2012. Circadian rhythms and depression: human psychopathology and animal models[J]. Neuropharmacology, 62(1): 101-114

Lee BH, Kim H, Park SH, Kim YK. 2007. Decreased plasma BDNF level in depressive patients[J]. J Affect Disord, 101(1-3): 239-244.

Le Gros Clark WE. 1924. On the brain of the tree shrew (Tupaia minor) $[\mathrm{M}] / /$ Proceedings of the Zoological Society of London. London: 
Academic Press, 1053-1074.

Legros C, Chalivoix S, Gabriel C, Mocaer E, Delagrange P, Fuchs E, Malpaux B. 2007. First evidence of melatonin receptors distribution in the suprachiasmatic nucleus of tree shrew brain[J]. Neuro Endocrinol Lett, 28(3): 267-273.

Manji HK, Drevets WC, Charney DS. 2001. The cellular neurobiology of depression[J]. Nat Med, 7(5): 541-547.

McTavish D, Benfield P. 1990. Clomipramine. An overview of its pharmacological properties and a review of its therapeutic use in obsessive compulsive disorder and panic disorder[J]. Drugs, 39(1): 136-153.

Meyer U, van Kampen M, Isovich E, Flügge G, Fuchs E. 2001. Chronic psychosocial stress regulates the expression of both GR and MR mRNA in the hippocampal formation of tree shrews[J]. Hippocampus, 11(3): 329-336

Naughton M, Mulrooney JB, Leonard BE. 2000. A review of the role of serotonin receptors in psychiatric disorders[J]. Hum Psychopharmacol, 15(6): 397-415.

Nikolarakis K, Pfeiffer A, Stalla GK, Herz A. 1987. The role of CRF in the release of $\mathrm{ACTH}$ by opiate agonists and antagonists in rats[ $\mathrm{J}]$. Brain Res, 421(1-2): 373-376.

Ohl F, Kirschbaum C, Fuchs E. 1999. Evaluation of hypothalamo-pituitary-adrenal activity in the tree shrew (Tupaia belangeri) via salivary cortisol measurement[J]. Lab Anim, 33(3): 269-274.

Pittenger C, Duman RS. 2008. Stress, depression, and neuroplasticity: a convergence of mechanisms[J]. Neuropsychopharmacology, 33(1): 88-109.

Santarelli L, Saxe M, Gross C, Surget A, Battaglia F, Dulawa S, Weisstaub N, Lee J, Duman R, Arancio O, Belzung C, Hen R. 2003. Requirement of hippocampal neurogenesis for the behavioral effects of antidepressants[J]. Science, 301(5634): 805-809.

Shen Y, Specht SM, De Saint Ghislain I, Li R. 2004. The hippocampus: a biological model for studying learning and memory[J]. Prog Neurobiol, 44(5): 485-496.

Shimizu E, Hashimoto K, Okamura N, Koike K, Komatsu N, Kumakiri C, Nakazato M, Watanabe H, Shinoda N, Okada S, Iyo M. 2003. Alterations of serum levels of brain-derived neurotrophic factor (BDNF) in depressed patients with or without antidepressants[J]. Biol Psychiatry, 54(1): 70-75.

Simon GE, Savarino J, Operskalski B, Wang PS. 2006. Suicide risk during antidepressant treatment[J]. Am J Psychiatry, 163(1): 41-47.

Skalisz LL, Beijamini V, Joca SL, Vital MA, Da Cunha C, Andreatini R. 2002. Evaluation of the face validity of reserpine administration as an animal model of depression-Parkinson's disease association[J]. Prog Neuropsychopharmacol Biol Psychiatry, 26(5): 879-883.

()Slotkin TA, Seidler FJ. 2006. Cholinergic receptor subtypes in the olfactory bulbectomy model of depression[J]. Brain Res Bull, 68(5): 341-345.

Spitzer RL, Gibbon ME, Skodol AE, Williams JBW. 1994. DSM-IV casebook: A learning companion to the Diagnostic and Statistical Manual of Mental Disorders 4th Ed[M]. Washington DC: American Psychiatric Association.

Túnez I, Drucker-Colín R, Montilla P, Peña J, Jimena I, Medina FJ, Tasset I. 2010. Protective effect of nicotine on oxidative and cell damage in rats with depression induced by olfactory bulbectomy[J]. Eur J Pharmacol, 627(1-3): 115-118.

van Kampen M, Kramer M, Hiemke C, Flügge G, Fuchs E. 2002. The chronic psychosocial stress paradigm in male tree shrews: evaluation of a novel animal model for depressive disorders[J]. Stress, 5(1): 37-46.

van Kampen M, Schmitt U, Hiemke C, Fuchs E. 2000. Diazepam has no beneficial effects on stress-induced behavioural and endocrine changes in male tree shrews[J]. Pharmacol Biochem Behav, 65(3): 539-546.

Vaugeois JM, El Yacoubi M, Costentin J. 2004. Comments on an animal model of depression[J]. Ann Pharm Fr, 62(5): 332-342.

Wang J, Zhou QX, Tian M, Yang YX, Xu L. 2011. Tree shrew models: a chronic social defeat model of depression and a one-trial captive conditioning model of learning and memory[J]. Zool Res, 32(1): 24-30.

Weigmann H, Härtter S, Hiemke C. 1998. Automated determination of clomipramine and its major metabolites in human and rat serum by high-performance liquid chromatography with on-line column-switching[J]. J Chromatogr B Biomed Sci Appl, 710(1-2): 227-233.

Willner P. 1991. Behavioural Models in Psychopharmacology: Theoretical Industrial, and Clinical Perspectives[M]. New York: Cambridge University Press.

Willner P. 2005. Chronic mild stress (CMS) revisited: consistency and behavioural-neurobiological concordance in the effects of CMS[J]. Neuropsychobiology, 52(2): 90-110.

Wilson N. 2002. Depression and its relation to light deprivation[J]. Psychoanal Rev, 89(4): 557-567.

Xu L. 2011. Animal models of human diseases[J]. Zool Res, 32(1): 1-3. [徐 林. 2011. 人类疾病的动物模型. 动物学研究, 32(1): 1-3.]

Zambello E, Fuchs E, Abumaria N, Rygula R, Domenici E, Caberlotto L. 2010. Chronic psychosocial stress alters NPY system: different effects in rat and tree shrew[J]. Prog Neuropsychopharmacol Biol Psychiatry, 34(1): 122-130 